\title{
Pharmaceutical Uses of Chitosan in the Medical Field
}

\author{
Alef Mustafa \\ Dona153 Pharmacy, Constanta, Romania \\ Emin Cadar \\ Minifarmpharmacy, Constanta, Romania
}

Rodica Sîrbu

"Ovidius" University of Constanţa, Faculty of Pharmacy,

Constanta, Romania

\begin{abstract}
Two of the considerably versatile and promising biomaterials are chitin and chitosan. Chitin is known to be the most abundant natural amino mucopolysaccharide, produced annually almost as much as cellulose, and it is found in the structure of a wide number of intervertebrates ( crustaceans' exoskeleton, insects' cuticles) functioning as a structural component that provides strenght and protection to the organisms, and the cell walls of fungi, among others. On the other hand, chitosan only occurs naturally in some fungi (mucoraceae). The composition of chitin is based on $\beta(1 \rightarrow 4)$-linked 2-acetamido-2-deoxy- $\beta$-D-glucose ( $\mathrm{N}$-acetylglucosamine). Due to their natural origin, both chitin and chitosan are defined as a family of polymers which present a high variability in their chemical and biological properties such as biocompatibility, biodegradability, mucoadhesion, anticholesterolemic, antitumoral, hemostatic and antimicrobial effect. These characteristics of chitin and chitosan have a major influence on the their properties and depending on the DD (degree of dezacetilation) and $\mathrm{Mw}$ (molecular weight), they are used in a variety of medical applications such ascosmetics, artificial skin, wound-dressings, water engeneering, opthalmology, drug-delivery system. The aim of this review is to highlight the physicochemical properties of chitin and chitosan used in the wound healing process.It is known that in the last years, the number of pacients suffering from wounds and burns difficult to treat and heal has increased. During the wound healing process, the dressing protects the injury and contributes to the recovery of dermal and epidermal tissues. Due to their high biocompatibility, biodegradability and similarity to the human body macromolecules these natural polysacharides (chitin and chitosan) are extensively used in wounds and burns management.
\end{abstract}

Keywords: chitosan, chitin, wound dressing, natural polymer, biomaterials

\section{Introduction}

A major interest in modern medicine is represented by the biometerials with marine origins. Among these, chitin and chitosan received special attention in the medical fields due to their unique properties.

Chitin and its deacetylated derivative, chitosan are natural polymers composed of randomly distributed $\beta$-(1-4)-linked Dglucosamine (deacetylated unit) and N-acetyl-D-glucosamine (acetylated unit). Both chitin and chitosan can not be defined as a unique chemical structure, but as a family of polymers, due to their natural origin, and also present a high variability in their chemical and physical properties. This variability is related not only to the origin of the samples but also to their method of preparation. So, a complete characterization of the samples is necessary.[1]

For chitin, there are known three crystalline forms: $\alpha-, \beta$-, andy-chitins. Chitosan is also crystalline and presents polymorphism depending on its physical state. The residual crystalinity may vary considerably depending on the origin of the polymer and its treatment during extraction from raw resources. 
In a recent study, the author, Rinaudo has reported that the origin of chitin influences not only its crystalinity and purity but also its polymer chain arrangement, and its properties. [2]. Chitin and chitosan are used in various field of medicine, such as biomedicine, food industry, agriculture and cosmetics. The success of use of chitosan in all of these specific applications is directly linked to the detailed research of their physico-chemical properties.

Chitin is known to be the most abundant natural amino mucopolysaccharide, produced annually almost as much as cellulose, and it is found in the structure of a wide number of intervertebrates

(crustaceans' exoskeleton, insects' cuticles) functioning as a structural component that provides strenght and protection to the organisms, and the cell walls of fungi, among others. On the other hand, chitosan only occurs naturally in some fungi (mucoraceae). The aim of this review is to highlight the physicochemical properties of chitin and chitosan used in the wound healing process.

\section{Research Methods}

The composition of chitin is based on $\beta(1 \rightarrow 4)$-linked 2-acetamido-2-deoxy- $\beta$-D-glucose ( $\mathrm{N}$-acetylglucosamine). Due to their natural origin, both chitin and chitosan are defined as a family of polymers which present a high variability in their chemical and biological properties such as biocompatibility, biodegradability, mucoadhesion, anticholesterolemic, antitumoral, hemostatic and antimicrobial effect.

The main parameters that affect the polymer properties are $\mathrm{DD}, \mathrm{Mw}$, polydispersity and crystalinity.

The purity (ash content), the moisture, the content of heavy metals,endotoxin and proteins must be determined for applications related to human consumption such as food and medical applications.

It has been demonstrated that the DD is one of the most important chemical characteristics, which could influence the performance of chitosan in many of its applications. The influence of average Mw on the viscosity development of aqueos solutions plays a significant role in the biochemical and biopharmacological significance of chitosan [3]. Due to its low solubility chitin $\mathrm{Mw}$ is not easily determined.

Table 1. shows the various methods for the determination of chitin and chitosan characteristics.[4]

Table.1 Physicochemical Characteristics of Chitin and Chitosan and the Determination Methods

\begin{tabular}{|l|l|}
\hline Physicochemical Characteristics & Determination Method \\
\hline DD & Infrared Spectroscopy \\
& UV- spectrophotometry \\
& $\begin{array}{l}\text { Nuclear magnetic resonance spectroscopy } \\
\text { Potentiometric titration }\end{array}$ \\
\hline Average Mw and/or Mw distribution & Viscosimetry \\
& Gel Permeation cromatography \\
\hline Moisture content & Gravimetric analysis \\
\hline Ash content & Gravimetric analysis \\
\hline Protein & Bradford method \\
\hline
\end{tabular}

As is shown in Table.1 different result are obtained when using methods based on different principles. In present, even the best characterized chitosans available on the market are usually described olny with regard to their average degree of acetylation and their average degree of polymerization (DP), their ash content and the absence of contaminating bacteria [5].

Apart from the specific characteristics, which are specific fosr each application, there is a degree of consensus regarding general characteristics that must be present in chitosan samples to be used in the field of biomedical applications: moisture content $\%$, ash content $\%$, protein content $\%$, insolubility $\%$, turbidity NTU units, DD \%.

For pharmaceutical applications, the chitosan requirements are: colour: white or slight yellow, particle size $<0.3 \mathrm{~mm}$, density $1.35-1.40 \mathrm{~g} / \mathrm{cm}^{3}, \mathrm{pH} 6.5-7.5$.

\section{Results and Discussions}


Chitin and chitosan are currently receiving a great deal of interest in the medical and pharmaceutical applications due to their interesting properties that make them suitable for use in the biomedical field, such as biocompatibility, biodegradability and non toxicity. Other properties such as analgesic, antitumor, hemostatic, hypocholesterolemic, antimicrobian and antioxidant properties have also been reported. A better understanding of the mechanism of these properties makes it necessary for chitosan to be well characterized and purified from accompanying compounds [6]. In addition chitin and chitosans derivatized in a variety of fashions can be used to prove molecular hypothesis for the biological activity. The parameter with a higher effect is the $\mathrm{DD}$, because the majority of the biological properties are related to the cationic behaviour of the chitosan. In some cases, the Mw has a predominant role. Beside the DD and Mw, other properties such as chain conformation, solubility or degree of substitution have been studied. Chitosans produced by heterogenous deacetylation, with a block arrangement of acetylated units, have a tendency to form aggregates in aqueous solutions.

Table. 2 shows the relationship between some chitin and chitosan biological properties and their physicochemical charateristics.[4]

Table.2. The relationship between some chitin and chitosan biological properties and their physicochemical charateristics

\begin{tabular}{|l|l|}
\hline Property & Characteristic \\
\hline Biodegradability & $\mathrm{DD}$, distribution of acetyl groups Mw \\
\hline Biocompatibility & $\mathrm{DD}$ \\
\hline Mucoadhesion & $\mathrm{DD}, \mathrm{Mw}$ (only chitosan) \\
\hline Hemostatic effect & $\mathrm{DD}, \mathrm{Mw}$ \\
\hline Analgesic effect & $\mathrm{DD}$ \\
\hline Adsorbtion enhancer & $\mathrm{DD}$ (only chitosan) \\
\hline Antimicrobian effect & $\mathrm{Mw}$ \\
\hline Anticolesterolemic effect & $\mathrm{DD}, \mathrm{Mw}$, viscozity \\
\hline Antioxidant effect & $\mathrm{DD}, \mathrm{Mw}$ \\
\hline
\end{tabular}

DD: deacetylation degree, Mw: molecular weight

\section{Biodegradability}

Chitin and chitosan are absent from mammals but they can be degraded in vivo by several proteases (lysozyme, papain, pepsin). Their biodegradation leads to the release of non-toxic oligosaccharides of variable length which can be subsenquently incorporated to glycosaminoglycans and glycoproteins, to metabolic pathways or be excreted. A degradation role on chitin and chitosan seems to play a non-specific protease present in all mammalian tissues- lysozyme. The lengths of the chains $(\mathrm{Mw})$ affects the degradation rate [7]. The understanding and control of the degradation rate of chitin and chitosan-based devices is of great interest since degradation is essential in many small and large molecule release

applications and in functional tissue regeneration applications. Ideally, the rate of scaffold degradation should mirror the rate of new tissue formation or be adequate for the controlled release of bioactive molecules. Thus, it is important to understand and control both the mechanism and the rate

by which each material is degraded. The degradation rate also affects the biocompatibility since very fast rates of degradation will produce an accumulation of the amino sugars and produce an inflammatory response.

Chitosan samples with low DD induce an acute inflammatory response while chitosan samples with high DD induce a minimal response due to the low degradation rate.Degradation has been shown to increase as DD decreases. Kofuji et al. investigated the enzymatic behaviours of various chitosans by observing changes in the viscosity of chitosan solution in the presence of lysozyme [8]. They found that chitosan with a low DD tended to be degraded more rapidly. It can be concluded that it is impossible to estimate biodegradation rate from the $\mathrm{DD}$ alone.

\section{Biocompatibility}

Both chitin and chitosan show very good compatibility but this property depends on the characteristics of the sample (natural source, method of preparation, Mw and DD). Due to its higher versatility and biological properties the majority of the assays have been carried out on chitosan samples. Although the gastrointestinal enzymes can partially degrade both 
chitin and chitosan, when both polymers are orally administered they are not absorbed. For this reason, they are considered as not bioavailable. Toxicity of chitosan is reported to depend on DD. It was reported that chitosans with DD higher than $35 \%$ showed low toxicity, while a DD under $35 \%$ caused dose dependant toxicity. On the other hand, Mw of chitosan did not influence toxicity. Residual proteins in chitin and chitosan could cause allergic reactions such as hypersensitivity. The protein content in a sample depends on the source of the sample and, especially, on the method of preparation.

\section{Hemostatic Effect}

Chitosan presents anticoagulant activity tested invitro [9]. The anticoagulant activity of chitosan seems to be related to its positive charge since red blood cells' membranes are negatively charged and chitin is less effective than chitosan. The hemostatic effect of chitosan is not related to the clasic coaulation pathways, but it can promote platelet agregation. The blood platelets play a very important role in the coagulation process and can lead to hemostatis and thrombosis. Besides platelets and erythrocytes, chitosan also accelerates thrombin generation.

\section{Analgezic Effect}

It was reported that both chitin and chitosan show analgesic effect. The analgesic effect of these biopolymers on inflammatory pain has been studied due to intraperitoneal administration of acetic acid. Chitosan showed a greater effect than chitin. This difference is due to the different action mechanism of the two polymers. It was demonstrated that themain analgesic effect of chitosan is the absorption of proton ions released in the inflammatory area.[4]

\section{Antitumor Activity}

An antitumor activity of chitosan has been claimed by inhibition of the growth of tumor cells mainly due to an immunevstimulation effect. chitosan oligomers possess antitumor activities tested both in vitro and in vivo [10].

Studies carried out using mice that had ingested low-Mw chitosan revealed significant antimetastatic effects of chitosan against Lewis lung carcinoma. Partially deacetylated chitin as well as chitin with a carboxymethyl group have also been effective to demote tumor progression. The suggested mechanism involves immunostimulating effects of chitin and its carboxymethyl derivatives via stimulation of cytolytic T-lymphocytes. This activity increases with smaller molecular sizes and it is suggested that they have immunostimulating effects that activate peritoneal macrophages and stimulate nonspecific host resistance. However, higher Mw oligomers have also exhibited antitumor activity. The effect of chitosan on tumor growth and metastasis was studied. The activation of macrophages by chitosan is suggested to mediate its antitumor effects in vivo, while its angiogenic inducing properties may be the harmful effects of chitosan, such as promotion of tumor growth and invasion [11].

\section{Anticholesterolemic Effect}

There are several proposed mechanisms for cholesterol reduction by chitosan. The entrapment caused by a viscous polysaccharide solution is thought to reduce the absorption of fat and cholesterol in the diet. On the other hand,

the presence of the amino group in its structure determines the electrostatic force between chitosan and anion substances, such as fatty acids and bile acids. The interaction between chitosan and anionic surfaceactive materials (phospholipids, bile acids) depends on its three types of reactive functional groups: the amino group at the C2 position and primary and secondary hydroxyl groups at the C-3 and C-6 positions, respectively. Although great effort has been made to find a correlation between the physicochemical characteristics of chitosan and its fat-binding capacity, only some significant relationships have been demonstrated [4].

\section{Antimicrobial Activity}

The antimicrobial activity of chitin, chitosan, and their derivatives against different groups of microorganisms, such as bacteria, yeast, and fungi, has received considerable attention in recent years. Two main mechanisms have been suggested as the cause of the inhibition of microbial cells bychitosan. The first mechanism refers to the interaction with anionic groups on the cell surface. Due to its polycationic nature, it causes the formation of an impermeable layer around the cell, which prevents the transport of essential solutes. Electron microscopy demostratedthat the site of action is the outer membrane of gram negative bacteria. The permeabilizing effect has been observed at slightly acidic conditions in which chitosan is protonated, but this permeabilizing effect of chitosan is reversible [12]. The second mechanism involves the inhibition of the RNA and protein synthesis by permeation into the cell nucleus. In this case the Mw is the decisive property [13] Other mechanisms have also been proposed. Chitosan may inhibit microbial growth by acting as a chelating 
agent rendering metals, trace elements or essential nutrients unavailable for the organism to grow at the normal rate. Chitosan is also able to interact with flocculate proteins, but this action is highly $\mathrm{pH}$-dependent.

\section{Antioxidative Activity}

Chitosan has shown a significant scavenging capacity against different radical species, the results being comparable to those obtained with commercial antioxidants. Samples prepared from crab shell chitin with DD of 90,75 and $50 \%$ where evaluated on the basis of their abilities to scavenge 1,1-diphenyl-2-picrylhydrazyl (DPPH) radical, hydroxyl radical, superoxide radical and alkyl radical. The results revealed that chitosan with higher DD exhibited the highest scavenging activity [14]. Chitosans of different size as well as their sulphate derivatives were assayed against superoxide and hydroxyl radicals. A negative correlation was found between chitosan $\mathrm{Mw}$ and activity. The chitosan sulphated derivatives presented a stronger scavenging effect on peroxide radicals but the chitosan of lowest Mw showed more considerable ferrous ionchelating potency than others [15]. The chelation of metal ions is one of the reasons why chitosan may be considered as a potential natural antioxidant for stabilizing lipid containing foods to prolong shelf life. Chitosans may retard lipid oxidation by chelating ferrous ions present in the system, thus eliminating their prooxidant activity or their conversion to ferric ion.

\section{Conclusion}

- Chitin and chitosan present a great variety of properties, allowing them to have a large number of applications.

- Chitosan, a natural polysaccahridic cation, received a considerable attention as a functional, non-toxic, reneweable and biodegradable polymer in a wide field of applications, especially in the pharmaceutical, cosmetic fields and food industry.

- In the medical field, chitosan was not only used as artificial skin and wound healing accelerator, but also as physiological material, due to its antitumor, imunomodulator, antimicrobial and anticholesterolemic properties.

- It was revealed that these characteristics depend on the chemical structure and molecular weight. In conclusion, the applications of this natural polysaccharide are limited by its high molecular weight (Mw) and its low solubility in non-acidic aqueous media.

\section{References}

[1] Helander I, Nurmiaho-Lassila E, Ahvenainen R, Rhoades J, Roller S. Chitosan disrupts the barrier properties of the outer membrane of Gram-negative bacteria. Int J Food Microbiol 2001; 71: 235-44.

[2] Huang M, Khor E, Lim L. Uptake and cytotoxicity of chitosan molecules and nanoparticles: effects of molecular weight and degree of deacetylation. Pharm Res 2004; 21 (2): 344-53

[3] Inmaculada Aranaz, Marian Mengibar, Ruth Harris. Functional Characterization of Chitin and Chitosan, Current Chem Biol, 2009,3 203-230

[4] Jeon YJ, Kim SK. Antitumor activity of chitosan oligosaccharides produced in ultrafiltration membrance reactor system. J Microbiol Biotechnol 2002; 12(3): 503-7.

[5] Kofuji K, Qian CJ, Nishimura M, Sugiyama I, Murata Y, Kawashima S. Relationship between physicochemical characteristics and functional properties of chitosan. Eur Polym J 2005; 41 (11): 2784-91.

[6] Kumar MNVR. A review of chitin and chitosan applications. React Funct Polym 2000; 46: 1-27

[7] Liu X, Yun L, Dong Z, Zhi L, Kang D. Antibacterial action of chitosan and carboxymethylated chitosan. J Appl Polym Sci 2001; 79(7): 1324-35.

[8] Moerschbacher B, El Gueddari N. Bio-activity matrices for partially acetylated chitosan oligomers. Advances in chitin Science vol IX (CD): 2007; 10-23

[9] Muzzarelli RAA, Muzzarelli C. Chitosan chemistry: Relevance to the biomedical sciences. Adv Polym Sci 2005; 186: 151-209.

[10] Park PJ, Je JY, Jung WK, Ahn CB, Kim SK. Anticoagulant activity of heterochitosans and their oligosaccharide sulfates. Eur Food Res Technol 2004; 219: 529-33.

[11] Park PJ, Je JY, Kim SK. Free radical scavenging activities of differently deacetylated chitosans using an ESR spectrometer. Carbohydr Polym 2004; 55(1):17-22. 
[12] Rinaudo M. Chitin and Chitosan. Properties and applications. Prog Polym Sci 2006; 31(7): 603-32

[13] Tharanathan RN, Kittur FS. Chitin: The Undisputed Biomecule of Great Potential. Crit Rev Food Sci Nut 2003; 43(1): 61-87

[14] Ueno H, Mori T, Fujinaga T. Topical formulations and wound healing applications of chitosan. Adv Drug Deliv Rev 2001; 52: 105-15.

[15] Xing R, Liu S, Guo Z, et al. Relevance of molecular weight of chitosan and its derivatives and their antioxidant activities in vitro. Bioorg Med Chem 2005; 13(5): 1573-7. 